\title{
Longer treatment duration with SLIT leads to higher patient satisfaction and clinical improvement. Outcomes of the SAMITES study
}

\author{
Enric Marti ${ }^{1}$, Carlos Sanchez-Salguero², Enrique Scorza ${ }^{1}$, Mario Alberto Garcia ${ }^{3}$ \\ From 9th Symposium of Experimental Rhinology and Immunology of the Nose (SERIN 2013) \\ Leuven, Belgium. 21-23 March 2013
}

\section{Background}

Sublingual immunotherapy (SLIT) is considered as a valid alternative in treatment of patients suffering house dust mite (HDM) allergic rhinitis. No evaluation of the patients' satisfaction on treatment and its relationship with clinical improvement and compliance was previously described.

\section{Methods}

An observational cross-sectional study was carried out to compare patients' satisfaction after 4-6 months (group A) or 9-12 months (group B) of SLIT treatment by the use of a validated Spanish satisfaction questionnaire (ESPIA) consisting of 16 items on a 5-point Likert scale (scale 16-80, higher score indicating more satisfaction). Secondary objectives were to investigate relation between satisfaction and both clinical improvement and compliance. Patients were classified in terms of compliance into <25\%; $25-50 \%, 51-75 \%$; $>76 \%$ group.

\section{Results}

Data from 232 patients (162 in A and 70 in B) were collected. In group A, $72 \%$ of them had persistent and 96\% moderate-severe rhinitis before starting immunotherapy. Similarly in group B, $68 \%$ of them had persistent and 97\% moderate-severe rhinitis. Treatment duration was $5.5 \pm 1.7$ months in A and $12.3 \pm 2.5$ months in B. Median total satisfaction was 60 and 73 points in $A$ and $B$ respectively. In those patients reporting compliance $>$ $76 \%(\mathrm{n}=191)$ higher median values of satisfaction (ESPIA score) were found in group $B(P<0.0001)$. Patients changing from persistent to intermittent rhinitis

${ }^{1}$ Clinica Internacional de Medicina Avanzada (CIMA), Allergy Service, Barcelona, Spain

Full list of author information is available at the end of the article between start of SLIT and current situation were $49 \%$ and $60 \%$ in A and B respectively. Concerning severity according to ARIA guidelines, patients changing from moderate-severe to mild were $43 \%$ and $72 \%$ in A and B respectively (group effect, $\mathrm{p}=0.0003$ ). In those patients reporting clinical improvement in terms of rhinitis frequency $(n=108)$ higher median values of satisfaction were found in group $B(P=0.0007)$ and similarly, patients reporting clinical improvement in terms of rhinitis severity $(n=151)$, higher median values of satisfaction were found in group $B(P<0.0001)$.

\section{Conclusions}

Almost $50 \%$ of the patients treated 5.5 months experienced an improvement of their rhinitis in terms of frequency (49\%) and severity (43\%), this percentage is higher in patients treated 12.3 months $60 \%$ and $72 \%$ respectively following ARIA classification. The SAMITES study demonstrates a clear relationship between patients' satisfaction with SLIT for HDM allergic rhinitis with the duration of treatment, compliance and clinical improvement.

\section{Author details}

${ }^{1}$ Clinica Internacional de Medicina Avanzada (CIMA), Allergy Service, Barcelona, Spain. ${ }^{2}$ Hospital Virgen de las Montañas, Allergy Service, Cadiz, Spain. ${ }^{3}$ Stallergenes Iberica, Medical Department, Barcelona, Spain.

Published: 16 July 2013

\section{doi:10.1186/2045-7022-3-S2-P12}

Cite this article as: Marti et al:: Longer treatment duration with SLIT leads to higher patient satisfaction and clinical improvement. Outcomes of the SAMITES study. Clinical and Translational Allergy 2013 3(Suppl 2): P12.
(C) 2013 Marti et al; licensee BioMed Central Ltd. This is an Open Access article distributed under the terms of the Creative Commons Attribution License (http://creativecommons.org/licenses/by/2.0), which permits unrestricted use, distribution, and reproduction in any medium, provided the original work is properly cited. 